\title{
PCDH10 gene inhibits cell proliferation and induces cell apoptosis by inhibiting the PI3K/Akt signaling pathway in hepatocellular carcinoma cells
}

\author{
MINGXIN YE, JINZHENG LI and JIANPING GONG \\ Department of Hepatobiliary Surgery, The Second Affiliated Hospital of Chongqing Medical University, \\ Chongqing 400010, P.R. China
}

Received November 8, 2016; Accepted January 4, 2017

DOI: $10.3892 /$ or.2017.5630

\begin{abstract}
Protocadherin10 (PCDH10), a member of the non-clustered protocadherin (PCDH) family, functions as a tumor-suppressor gene in many cancers. Previous studies have demonstrated that the expression of PCDH10 was noticeably downregulated in the tissue and cells of hepatocellular carcinoma (HCC), when compared to those in normal liver tissue. The decreased PCDH10 expression in HCC was correlated with the aberrant methylation status of PCDH10 promoter. However, the biological functions and molecular mechanism of PCDH10 in HCC have yet to be elucidated. The aim of the present study was to identify the biological function and mechanisms of PCDH10 in HCC. Quantitative real-time polymerase chain reaction was used to detect the expression of PCDH10 in HCC cells with decreased expression of PCDH10 which were transfected with plasmid pcDNA3.1-PCDH10 or pcDNA3.1-vector using Lipofectamine 2000. The biological effects of PCDH10 in HCC cells were detected by CCK-8, colony formation and flow cytometric assays. Western blot and co-immunoprecipitation (Co-IP) assays were performed to explore the mechanism of PCDH10 in HCC cells. PCDH10 expression was downregulated in the HCC cells (HepG2, $\mathrm{HuH7}$, HuH1, and SNU387) when compared to the normal liver cells (L02). Upregulation of PCDH10 inhibited cell proliferation and induced cell apoptosis in the HCC cells. More importantly, we revealed that PCDH10 inhibited the PI3K/Akt signaling pathway thus carrying out its suppressive function in HCC. This study provides insights into the tumorigenesis and progression of $\mathrm{HCC}$, and puts forward the novel hypothesis that PCDH10 could be a new biomarker for $\mathrm{HCC}$, or that combined with other molecular markers could
\end{abstract}

Correspondence to: Dr Jinzheng Li or Professor Jianping Gong, Department of Hepatobiliary Surgery, The Second Affiliated Hospital of Chongqing Medical University, 76 Linjiang Road, Chongqing 400010, P.R. China

E-mail: lijinzhengcqmu@163.com

E-mail: doctor115@126.com

Key words: PCDH10, hepatocellular carcinoma, proliferation, apoptosis, PI3K/Akt signaling pathway increase the specificity and sensitivity of diagnostic tests for HCC. Restoration of PCDH10 could be a valuable therapeutic target for $\mathrm{HCC}$.

\section{Introduction}

Hepatocellular carcinoma (HCC), a primary malignancy of the liver, is one of the most prevalent cancers, with an increasing incidence and mortality rate around the world $(1,2)$. The most effective therapy is liver resection or transplantation for patients with early-stage disease, however, most patients are diagnosed in later or inoperable stages (3). Although the diagnosis and therapies for HCC have advanced in recent years, the prognosis for HCC patients remains poor $(4,5)$. Therefore, it is imperative to clarify the molecular mechanisms underlying HCC, and to discover valuable diagnostic and prognostic biomarkers for HCC. Furthermore, new therapeutic agents to treat this malignancy must be explored.

Cadherin is a calcium-dependent adhesion protein that is a member of a large family of cell adhesion molecules. Cadherins have been identified by the presence of extracellular cadherin repeats of about 110 amino acid residues, and can be classified into: the classical cadherins, desmosomal cadherins, and protocadherins (PCDHs) (6,7). PCDHs are predominantly expressed in the nervous system, and are reported to participate in the circuit formation and maintenance of the brain $(8,9)$. However, in past decades accumulating evidence has revealed that $\mathrm{PCDH}$ family members act as tumor-suppressor genes in multiple carcinomas (10-14).

The protocadherin10 (PCDH10) gene is located on human chromosome $4 \mathrm{q} 28.3$. The PCDH10 protein belongs to the PCDH subfamily, and is expressed on the plasma membrane. Previous research regarding PCDH10 focused on neuronal diseases, such as autism (15). However, recent studies have demonstrated that PCDH10 is frequently downregulated by promoter DNA methylation, and functions as a tumorsuppressor gene in gastric, colorectal and lung cancer, as well as in many other carcinomas (16-19). Previous studies have indicated that the expression of PCDH10 was notably downregulated in HCC tissue and cells, compared to that in normal liver tissue (20). Furthermore, decreased PCDH10 expression was found to correlate with the methylation status of the 
PCDH10 promoter (20). However, the biological functions and mechanism of PCDH10 in HCC have yet to be elucidated. Therefore, the aim of the present study was to identify the biological function and molecular mechanism of PCDH10 in HCC, thus aiding the discovery of valuable diagnostic and prognostic biomarkers for $\mathrm{HCC}$, as well as the development of new therapeutic agents to treat this malignancy.

\section{Materials and methods}

Cell culture and transfection. HCC cell lines (HepG2, HuH7, HuH1 and SNU387) and a normal liver cell line (L02) were purchased from the American Type Culture Collection (ATCC; Mannasas, VA, USA). The cells were cultured in Dulbecco's modified Eagle's medium (DMEM; Hyclone Laboratories, Inc., Logan, UT, USA) with 10\% fetal bovine serum (FBS; Gibco, Grand Island, NY, USA). All the cells were maintained at $37^{\circ} \mathrm{C}$ in an incubator with $95 \%$ air and $5 \% \mathrm{CO}_{2}$.

The plasmid pcDNA3.1-PCDH10 and pcDNA3.1-vector were purchased from GeneChem Co., Ltd. (Shanghai, China). The transfection was performed in 6-well plates. Cells (HepG2 and HuH7) were seeded into 6-well plates and allowed to culture overnight. The wells were then filled with $1 \mathrm{ml}$ of fresh, serum-free medium after washing the cells twice with serum-free medium. Four micrograms of plasmid (pcDNA3.1-PCDH10 or pcDNA3.1-vector) and $5 \mu \mathrm{l}$ of Lipofectamine 2000 (Invitrogen, Carlsbad, CA, USA) were diluted in $500 \mu 1$ of serum-free medium respectively, and allowed to incubate for $5 \mathrm{~min}$ at room temperature. Following this, plasmid and Lipofectamine 2000 diluent were mixed and incubated for $20 \mathrm{~min}$ at room temperature, then $1 \mathrm{ml}$ of the aforementioned mixture was added to each well. Renewal of the medium with $2 \mathrm{ml}$ of fresh medium with $10 \%$ FBS was conducted after culturing $4 \mathrm{~h}$ at $37^{\circ} \mathrm{C}$. The efficiency of transfection was detected with RT-qPCR and western blotting assays.

Reverse transcription-quantitative polymerase chain reaction (RT-qPCR). TRIzol (Invitrogen) was used to isolate the total RNA from cells. The concentration and purity of total RNA were assessed, with absorbance ratio $\mathrm{OD}(260) / \mathrm{OD}(280)$ values with a purity range of 1.8-2.0. Total RNA was reverse-transcribed into cDNA using the GoScript reverse transcription kit (Promega, Madison, WI, USA). RT-qPCR amplification primers that were synthesized by Takara Bio (Dalian, China) are as follows: target gene PCDH10 forward, 5'-ACTGCTAT CAGGTATGCCTG-3' and reverse, 5'-GTCTGTCAACTAGA TAGCTG-3'; internal control $\beta$-actin forward, 5'-CTCCATC CTGGCCTCGCTGT-3' and reverse, 5'-GCTGTCACCTT CACCGTTCC-3'. RT-qPCR was performed with the SYBR ${ }^{\circledR}$ Premix ExTaq ${ }^{\mathrm{TM}}$ II (Tli RNaseH Plus; Takara Biotechnology Co., Ltd.) and according to the manufacturer's protocol on the Light-cycler $480^{\circledR}$ II real-time PCR system (Roche Diagnostics, Basel, Switzerland). The $2^{-\Delta \Delta C t}$ method was used to determine the relative quantitation of gene expression levels.

Western blot assay. Cells were scraped and collected after washing with phosphate-buffered saline (PBS). RIPA lysate buffer (Beyotime Institute of Biotechnology, Shanghai, China) was used to extract total protein, and the concentration of the protein was assessed using a BCA kit (Beyotime Institute of Biotechnology). Proteins were separated by polyacrylamide gel electrophoresis, transferred onto polyvinylidene fluoride (PVDF) membranes (Beyotime Institute of Biotechnology), blocked with 5\% skim milk for $2 \mathrm{~h}$ at room temperature, and then incubated with primary antibodies for PCDH10 [mouse anti-human monoclonal antibody (Abnova, Taipei, Taiwan)] and $\beta$-actin [mouse anti-human monoclonal antibody (Cell Signaling Technology, Inc., Danvers, MA, USA)] at $4^{\circ} \mathrm{C}$ overnight. Subsequently, the membranes were washed with TBST buffer three times, and then incubated with the secondary antibodies (goat anti-mouse; Cell Signaling Technology) with horseradish peroxidase (HRP) for $2 \mathrm{~h}$ at room temperature. The blots were washed again and detected with BeyoECL Plus kits (Beyotime Institute of Biotechnology). Fusion software was used to analyze the results.

Cell proliferation assay. Cell Counting Kit-8 (CCK-8; Dojindo) was used to evaluate cell proliferation. Cells (HepG2 and $\mathrm{HuH7}$ ) were seeded in a 96-well plate with a density of $5 \times 10^{3}$ cells/well, allowed to culture for $24 \mathrm{~h}$, and then transfected with plasmid pcDNA3.1-PCDH10 or pcDNA3.1-vector as previously described. According to the manufacturer's protocol, the CCK- 8 reagent was added to each plate at 0 , 24,48 and $72 \mathrm{~h}$ after transfection. Subsequently, the reaction was incubated for $2 \mathrm{~h}$ in the incubator, and then the OD was detected at $450 \mathrm{~nm}$ generating a cell proliferation curve.

Clone formation assay. Cells (HepG2 and $\mathrm{HuH} 7)$ were seeded in a 6-well plate and transfected with plasmid pcDNA3.1-PCDH10 or pcDNA3.1-vector. Approximately $48 \mathrm{~h}$ post-transfection, all cells were collected and counted, seeded in a 6-well plate at a density of $1 \times 10^{4}$ cells/well, and cultured under selectiong by G418 $(0.6 \mathrm{mg} / \mathrm{ml})$ for more than 2 weeks. The surviving clones ( $\geq 50$ cells) were counted after fixation with paraformaldehyde and staining with Gentian violet.

Flow cytometric assays of the cell cycle and cell apoptosis. For the cell cycle assay, $\mathrm{HepG} 2$ and $\mathrm{HuH} 7$ cells were seeded into 6-well plates and transfected with plasmid pcDNA3.1-PCDH10 or pcDNA3.1-vector. After incubation with transfection media for $24 \mathrm{~h}$, the cells were washed with PBS, digested by trypsin without EDTA, then collected and fixed in precooling $70 \%$ ethanol overnight at $4^{\circ} \mathrm{C}$. The cells were then washed with precooling PBS and then stained with propidium iodide (PI) $50 \mathrm{mg} / \mathrm{ml}$ for $30 \mathrm{~min}$ at $4^{\circ} \mathrm{C}$ in dark. Elite ESP flow cytometry was performed to assay the cell-cycle profiles. Data were analyzed with CELL Quest software (BD Biosciences, San Jose, CA).

The apoptosis of HepG2 and HuH7 cells was assessed by flow cytometry, combined with Annexin V-FITC/PI staining. The cells were transfected as previously described, and $48 \mathrm{~h}$ after transfection the cells were gently collected, and then incubated with Annexin V-fluorescein isothiocynate and PI at room temperature. The samples were evaluated by flow cytometry immediately.

Molecular mechanism of PCDH1O in HCC cells. PCDH10 plays an important role in cell-cell signal conduction, and previous studies have demonstrated that PCDH10 could regulate pathways 


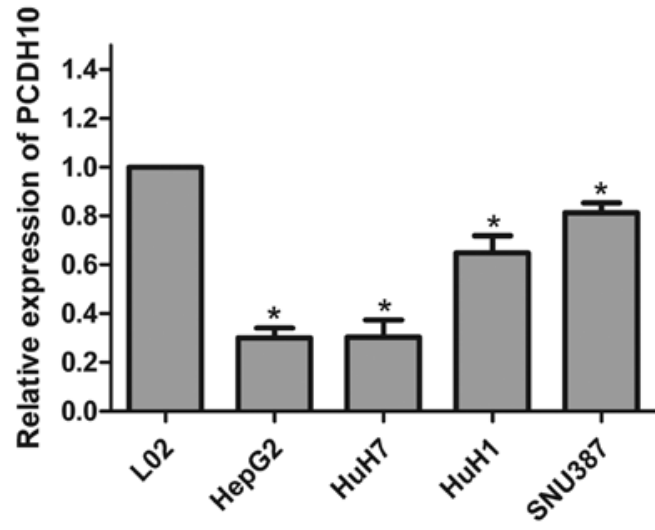

Figure 1. PCDH10 expression in $\mathrm{HCC}$ cells $(\mathrm{HepG} 2, \mathrm{HuH} 7, \mathrm{HuH} 1$ and SNU387) and normal liver cells (L02) detected with RT-qPCR. "P<0.05 compared to normal liver cells (L02). PCDH10, protocadherin10; RT-qPCR, reverse transcription-quantitative polymerase chain reaction.
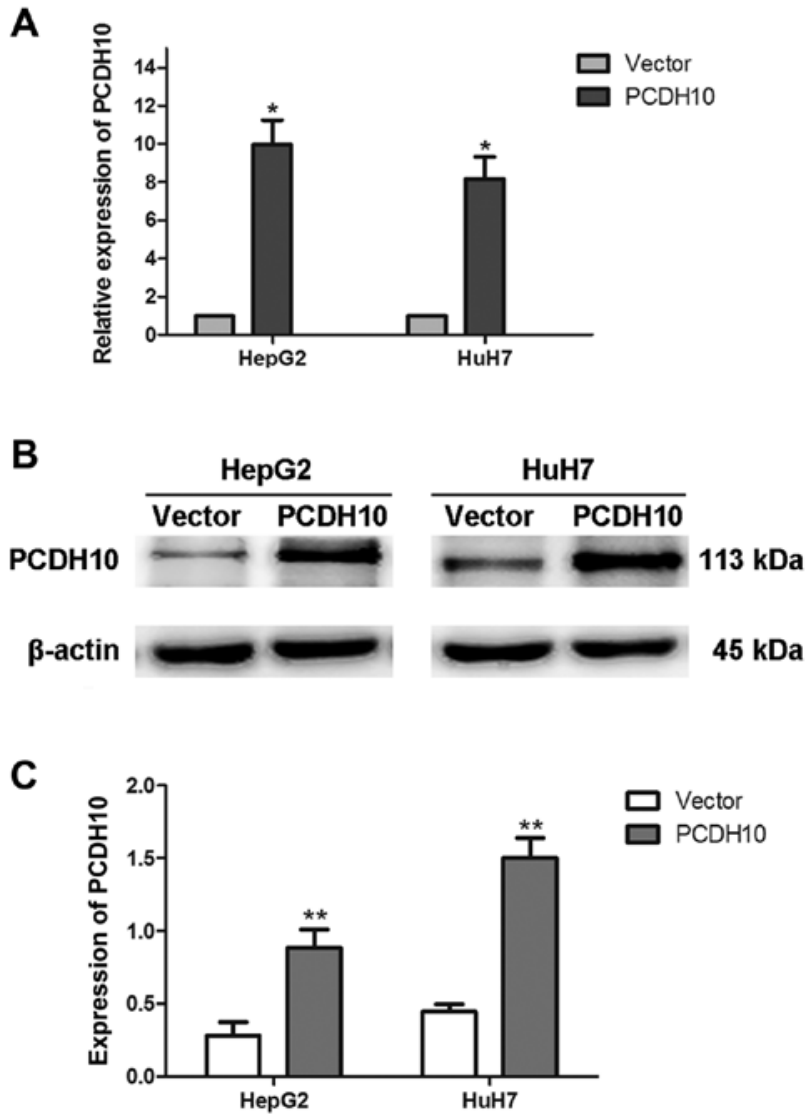

Figure 2. PCDH10 expression is significantly increased in cells transfected with PCDH10. (A) PCDH10 expression was assessed with RT-qPCR. (B and C) PCDH10 expression was assessed with western blot analysis and quantitative analysis. PCDH10: cells transfected with plasmid PCDH10 vector: cells transfected with the plasmid vector, ${ }^{*} \mathrm{P}<0.05,{ }^{* *} \mathrm{P}<0.01$ compared to the vector. PCDH10, protocadherin10; RT-qPCR, reverse transcription-quantitative polymerase chain reaction.

relating to tumorigenesis and tumor progression $(27,28)$. In this study, we observed a difference of phenotype in HCC cells after overexpression of PCDH10. Therefore, we speculated that the signaling pathway could regulate cell proliferation and cell apoptosis. Western blot analyses were performed to detect the target proteins. The western blot analysis method was aforementioned. Briefly, 48-72 h post-transfection, total proteins were extracted, separated by polyacrylamide gel electrophoresis, and then transferred to PVDF membranes. The membranes were blocked with $5 \%$ skim milk and then incubated with primary antibodies for p-AKT, p-MDM2, p53, p-GSK-3 $\beta$ and p21 (Abcam, Cambridge, MA, USA) and caspase-3, Bax, Bcl-2 and cyclin D1 (Cell Signaling Technology, Inc.) overnight at $4^{\circ} \mathrm{C}$. Membranes were then incubated with HRP-conjugated secondary antibodies, and then the blots were visualized with BeyoECL Plus kits.

To further explore how PCDH10 affects signaling pathways, we performed co-immunoprecipitation (Co-IP) assays to detect the molecules interacting with PCDH10. Co-IP assays were performed with the Co-IP kit (Life Technologies, Carlsbad, CA, USA) according to the manufacturer's protocol. HepG2 cells were transfected with plasmid pcDNA3.1-PCDH10. Approximately $48 \mathrm{~h}$ post-transfection, total proteins were extracted from the cells, and a PCDH10antibody, a phosphoinositide 3-kinase (PI3K) p85-antibody [mouse monoclonal antibody (Abcam)] and a control mouse antibody $\lg \mathrm{G}$ (Abcam) were used to perform immunoprecipitations. Samples were analyzed by western blot analysis as aforementioned.

Statistical analysis. Data are presented as the mean \pm SD from independent triplicate experiments, and analyzed with Student's t-test. $\mathrm{P}<0.05$ was considered to indicate a statistically significant result. All data analysis was performed with SPSS version 18.0 (IBM, Armonk, NY, USA).

\section{Results}

PCDH10 expression is dysregulated in HCC cells. We used RT-qPCR to detect the expression of PCDH10 in HCC cells and normal liver cells. The expression of PCDH10 was significantly less in the HCC cells when compared to that in the normal liver cells (L02) $(\mathrm{P}<0.05$; Fig. 1).

Efficiency of transfection in HCC cells, HepG2 and HuH7. HepG2 and HuH7 were transfected with plasmid pcDNA3.1-PCDH10 or pcDNA3.1-vector. Approximately $48 \mathrm{~h}$ later, the total RNA and proteins were extracted, and RT-qPCR and western blot analysis assays were used to assess the efficiency of transfection. The results indicated that PCDH10 expression was increased in the cells transfected with plasmid pcDNA3.1-PCDH10 when compared to that in the cells transfected with the pcDNA3.1-vector $(\mathrm{P}<0.05$; Fig. 2$)$.

PCDH10 inhibits cell proliferation of HCC. To assess the influence of PCDH10 on cell proliferation in HCC, the CCK-8 assay was performed. Cells were transfected with plasmid PCDH10 or vector and monitored for proliferation. The proliferation ability of the PCDH10-transfected cells was lower than that of the vector-transfected cells at 24,48 and $72 \mathrm{~h}(\mathrm{P}<0.05$; Fig. 3).

PCDH10 inhibits clone formation in HCC cells. The clone formation assay was performed to evaluate the suppressive effect of PCDH10 in HCC cells. As we expected, there was a strong decrease in the number of clones, and a significant decrease in 

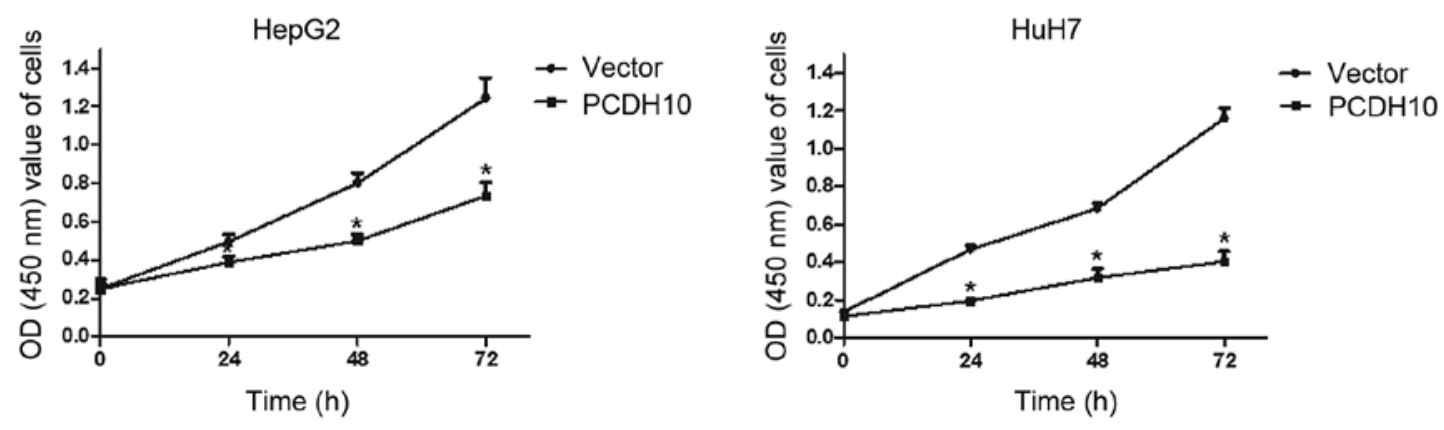

Figure 3. Effect of PCDH10 on cell proliferation in HCC. PCDH10: cells transfected with plasmid PCDH10; vector: cells transfected with the plasmid vector, ${ }^{*} \mathrm{P}<0.05$ compared to the vector. $\mathrm{PCDH} 10$, protocadherin10; HCC, hepatocellular carcinoma.

A
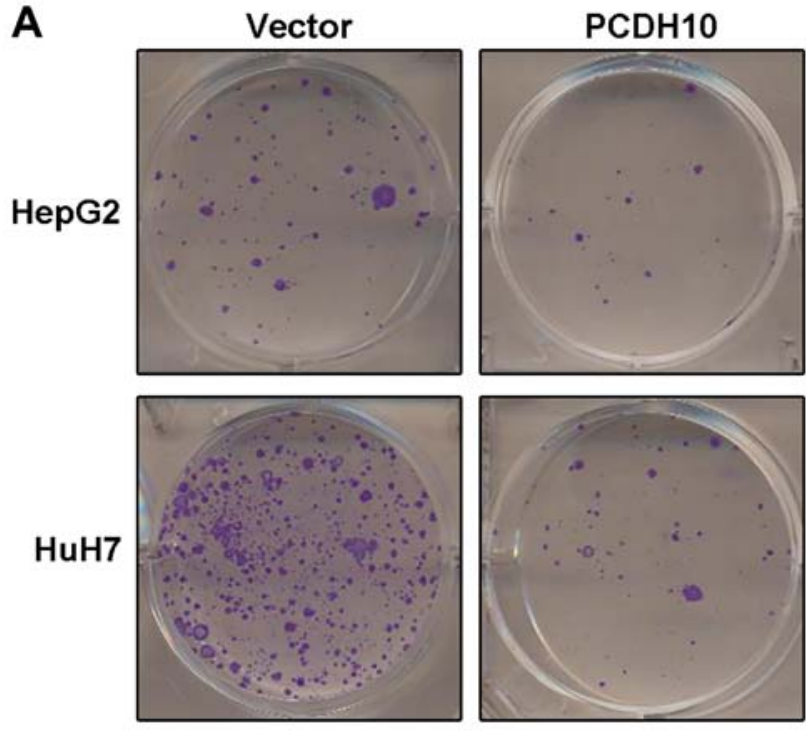

B

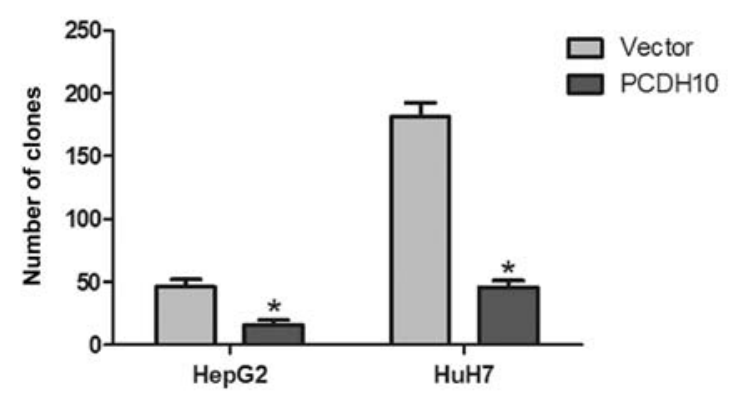

Figure 4. Effect of PCDH10 on cell clone formation in HCC. (A) PCDH10 significantly inhibits cell clone formation in HCC cells. (B) The quantitative analysis of the clone formation assay. PCDH10: cells transfected with plasmid PCDH10; vector: cells transfected with the plasmid vector, ${ }^{*} \mathrm{P}<0.05$ compared to the vector. PCDH10, protocadherin10; HCC, hepatocellular carcinoma.

the size of cells transfected with PCDH10 when compared to the cells transfected with the vector $(\mathrm{P}<0.05$; Fig. 4).

PCDH1O arrests the cell cycle at the G1 phase and induces cell apoptosis in HCC. Cell proliferation and clone formation assays were performed and it was determined that PCDH10 could inhibit HCC cell growth. In order to further understand how PCDH10 restricts cell growth, we used flow cytometry to analyze the cell cycle and cell apoptosis. The results indi- cated that in cells transfected with PCDH10, the number of cells in the G1 phase was increased, and the number of cells in the G2 phase were decreased, as compared to the control groups transfected with the vector $(\mathrm{P}<0.05$; Fig. 5). Notably, flow cytometry combined with Annexin V-FITC/PI staining was used to assess cell apoptosis, and these results indicated that cell apoptosis was markedly increased in the cells transfected with PCDH10, when compared to the control groups $(\mathrm{P}<0.05$; Fig. 6).

PCDH1O alters the PI3K/Akt signaling pathway in HCC cells. Dysregulation of the PI3K/Akt signaling pathway is implicated in a number of human diseases, including cancer. The PI3K/Akt pathway can promote cell proliferation and inhibit cell apoptosis via a series of complicated phosphorylation reactions. In this study, western blot analyses were performed to detect changes in the PI3K/Akt signaling pathway after transfecting PCDH10 into HepG2 HCC cells. We demonstrated that the protein levels of p-Akt, p-MDM2, p-GSK-3 $\beta$, $\mathrm{Bcl}-2$, and cyclin D1 were decreased, while caspase-3, p53, p21, and Bax were increased, in cells transfected with PCDH10 as compared to the control groups $(\mathrm{P}<0.05$; Fig. $7 \mathrm{~A}$ and $\mathrm{B})$. Our results suggested that the Akt signaling pathway was impaired. To explore how PCDH10 regulates the pathway, we hypothesized that PCDH10 could affect the proteins upstream of Akt. Fortunately, the Co-IP assay demonstrated interaction between PCDH10 and PI3K (Fig. 7C). These results indicate that PCDH10 inhibits cell proliferation and induces cell apoptosis via suppression of the PI3K/Akt signaling pathway in HCC cells (Fig. 7D).

\section{Discussion}

PCDH10 belongs to the PCDH family, a subfamily of the cadherin superfamily. This family member contains 6 extracellular cadherin domains, a transmembrane domain, and a cytoplasmic tail differing from those of the classical cadherins. The encoded protein of PCDH10 is a cadherin-related neuronal receptor thought to function in the establishment of specific cell-cell connections in the brain. Although PCDH10 is mainly expressed in the brain, it can be found in other organs, and a multitude of epigenetic studies have found that the PCDH10 promoter has high DNA methylation, which results in silencing of gene expression in many tumors. The methylation status of PCDH10 could 
A

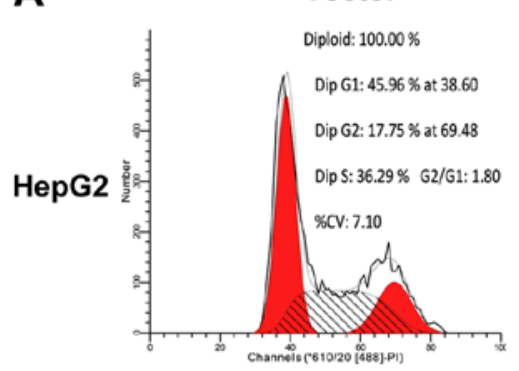

C

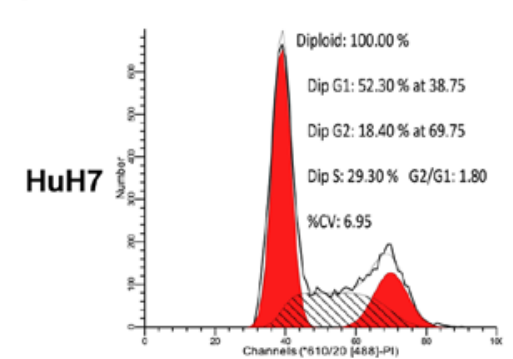

PCDH10

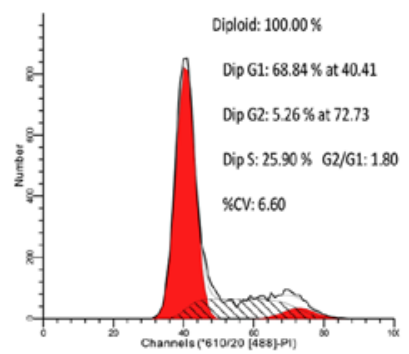

PCDH10

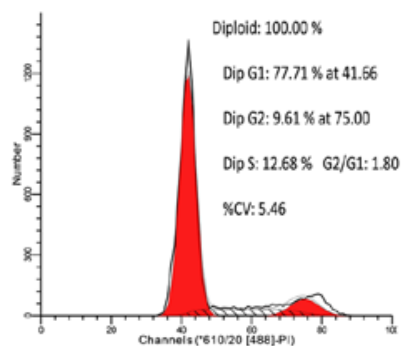

B

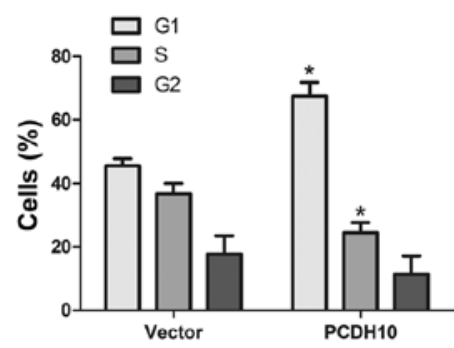

D

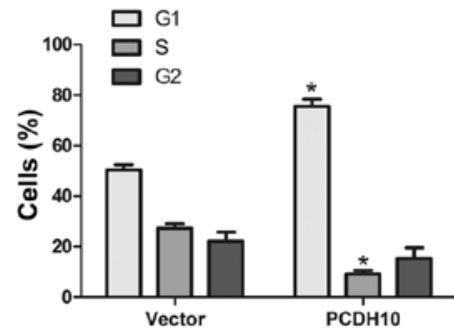

Figure 5. Effect of PCDH10 on the cell cycle. (A and C) PCDH10 arrested the cell cycle at the G1 phase in HepG2 and HuH7 cells as assessed with flow cytometry. (B and D) The quantitative analysis of the cell cycle assay in HepG2 and HuH7 cells. PCDH10: cells transfected with plasmid PCDH10; vector: cells transfected with the plasmid vector, ${ }^{\mathrm{P}}<0.05$ compared to the vector. $\mathrm{PCDH} 10$, protocadherin 10.

A
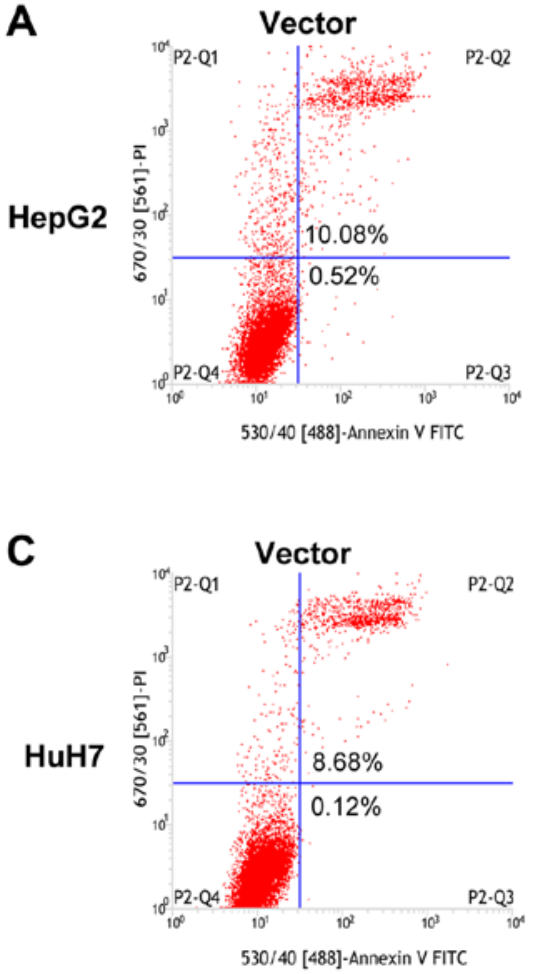

PCDH10
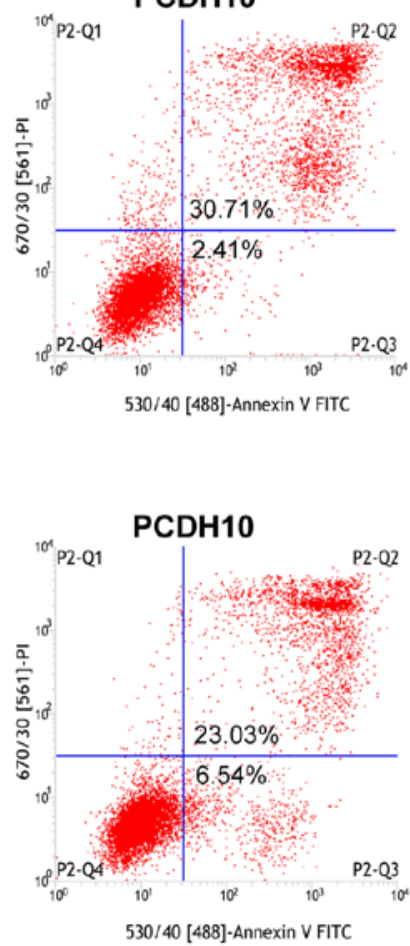

B

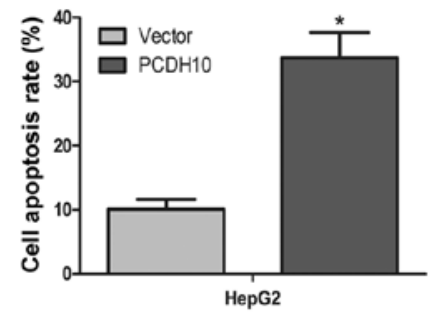

D

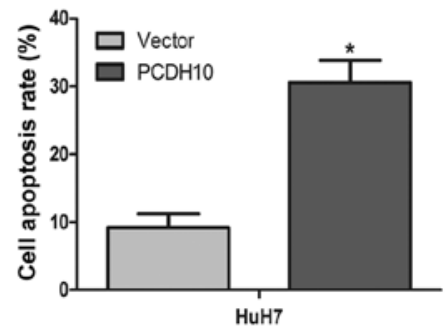

Figure 6. Effect of PCDH10 on cell apoptosis. (A and C) PCDH10 induces cell apoptosis in HepG2 and HuH7 cells as assessed with flow cytometry combined with Annexin V-FITC/PI staining. (B and D) The quantitative analysis of the cell apoptosis assay in HepG2 and HuH7 cells. PCDH10: cells transfected with plasmid PCDH10; vector: cells transfected with the plasmid vector, ${ }^{*} \mathrm{P}<0.05$ compared to the vector. PCDH10, protocadherin 10.

predict the prognosis of cancers (21-23). Further studies have demonstrated that re-expression of PCDH10 inhibited cellular proliferation, invasion ability, and increased cell apoptosis in multiple cancers $(19,22,24)$. These studies demonstrated that PCDH10 plays an important role in tumorigenesis and tumor development. Previous studies demonstrated that the expression of PCDH10 was notably downregulated in HCC tissues and cells when compared to normal liver tissue, and decreased PCDH10 expression in HCC was correlated with the methylation status of the PCDH10 promoter (20). However, 
A

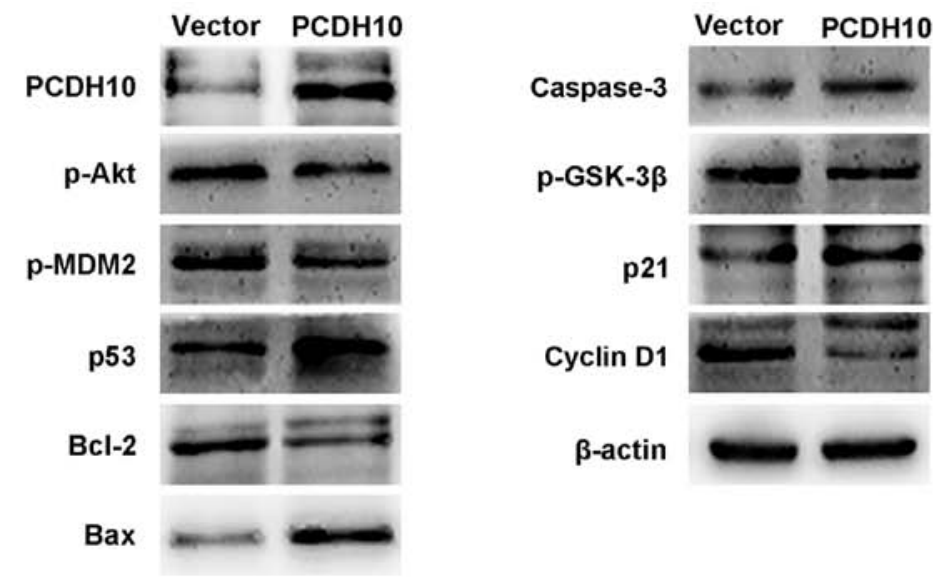

B
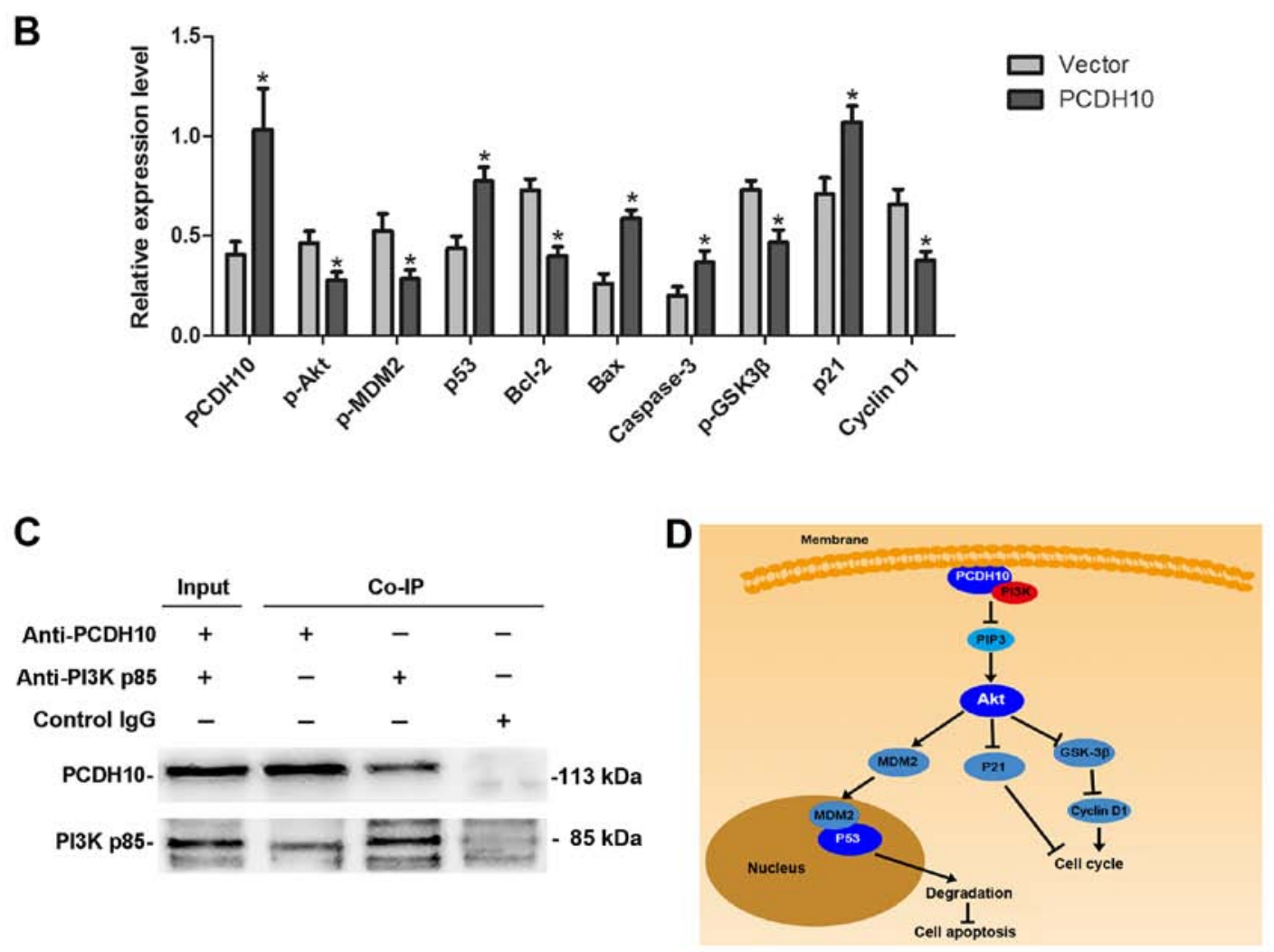

Figure 7. PCDH10 inhibits the PI3K/Akt signaling pathway. (A) The expression level of proteins in the Akt signaling pathway examined with western blot analysis. (B) The quantitative analysis of the expression level. (C) Co-IP assay indicates the interaction between PCDH10 and PI3K. (D) The network displaying the inhibition of the PI3K/Akt signaling pathway by PCDH10. PCDH10: cells transfected with plasmid PCDH10; vector: cells transfected with the plasmid vector, "P<0.05 compared to the vector. PCDH10, protocadherin10; PI3K, phosphoinositide 3-kinase; Co-IP, co-immunoprecipitation.

the role of PCDH10 in HCC has not yet been elucidated. In this study, we restored PCDH10 expression in HCC cells and observed that PCDH10 inhibited cell growth of HCC. This effect occured through cell cycle arrest at the G1 phase and induction of apoptosis of HCC cells. When we assessed the expression levels of the cell cycle-related protein cyclin D1 and the apoptosis-related protein caspase- 3 , the amount of cyclin D1 protein was decreased and the amount of caspase-3 protein was increased. This indicates that PCDH10 inhibits HCC cellular proliferation via cell cycle arrest and induction of apoptosis. Combined with results from previous studies, we propose that PCDH10 is a tumor-suppressor gene frequently downregulated with promoter methylation in $\mathrm{HCC}$, which is consistent with the role of PCDH10 in other cancers.
PCDHs have been identified as regulators of other molecules. PCDHs lack a cytoplasmic $\beta$-catenin binding site present in classical cadherins. The cytoplasmic domains of non-clustered PCDHs are different from each other, and their homology ranges from low to moderate $(7,9)$. Therefore, non-clustered PCDHs could act as regulators via interaction with a variety of intracellular binding partners. It has been reported that PCDH10/OL-PC interacts with Nck-associated protein 1 (Nap1)/WAVE1 and that the PCDH10/Nap1/WAVE1 complex affects actin assembly and subsequently regulates cell migration $(25,26)$. Recent studies demonstrated that PCDH10 is involved in some signaling pathways, including the nuclear factor- $\mathrm{\kappa B}$ signaling pathway and the $\mathrm{Wnt} / \beta$-catenin/BCL-9 signaling pathway $(27,28)$. These results demonstrate that 
PCDH10 carries out the suppressive function via interference with the signaling pathways that are involved in malignancy.

The Akt signaling pathway has become a major focal point because of its critical role in the regulation of diverse cellular functions including metabolism, growth, proliferation, survival, transcription, and protein synthesis. Dysregulation of the Akt signaling pathway can result in many diseases, including cancers (29). It is well known that the Akt signaling pathway is activated by receptor tyrosine kinases, integrins, B and T cell receptors, cytokine receptors, $\mathrm{G}$-protein-coupled receptors, and other stimuli that induce production of phosphatidylinositol $(3,4,5)$-trisphosphates (PIP3) by PI3K, and PIP3 via PDK1 phosphorylating Akt at Thr308 leading to partial activation of Akt. Activated Akt contributes to cell proliferation via phosphorylation of the CDK inhibitor $\mathrm{p} 21$, or by inhibiting the activity of GSK-3 $\beta$ by phosphorylation, and GSK-3 $\beta$ can regulate cyclin D1 proteolysis and subcellular localization. Akt inhibits cell apoptosis through the phosphorylation of the downstream molecule MDM2. Phosphorylation of MDM2 mediates ubiquitination and degradation of p53, a well-known molecule with various biological functions (30,31). In our study, we demonstrated that PCDH10 inhibited HCC cell proliferation and increased cell apoptosis, and in view of the research on PCDH10 in other tumors $(19,28)$, we put forward the hypothesis that PCDH10 may regulate the Akt signaling pathway in HCC. We detected the Akt signaling pathway and found that the protein levels of p-Akt, p-MDM2, Bcl-2 and cyclin D1 were decreased while p53, p-GSK3 $\beta$, p21, Bax and caspase-3 were increased in cells transfected with PCDH10 when compared to the control groups. This evidence indicates that PCDH10 can inhibite the Akt signaling pathway in HCC.

To illuminate the mechanism by which $\mathrm{PCDH} 10$ regulates the Akt signaling pathway, we detected the level of PI3K, a key upstream molecule of Akt. However, we found that the protein level of PI3K exhibited no significant change after PCDH10 overexpression (data not shown). The Co-IP assays demonstrated that there was an interaction between PCDH10 and PI3K. As previously described, PI3K, a phosphoinositide-kinase, catalyzes the production of phosphatidylinositol $(3,4,5)$-triphosphate (PIP3) which is necessary for the activation of $\mathrm{Akt}$, and this phosphorylation event is triggered by growth factors and hormones. We inferred that PCDH10 acts as a competitive substrate binding with PI3K to obstruct the catalytic reaction of PIP3 synthesis. Consequently, the activation of Akt is inhibited.

In summary, in the present sutdy, we explored the function and mechanism of PCDH10 in HCC cells. Our results indicated that PCDH10 can inhibit cell proliferation and induce cell apoptosis by negative regulation of the PI3K/Akt signaling pathway. Combined with the results of previous research, we conclude that PCDH10 acts as a tumor-suppressor gene in HCC by inhibiting the PI3K/Akt signaling pathway. This helps us to better understand the tumorigenesis and progression of HCC. Notably, these experiments provide novel insights and put forward the hypothesis that PCDH10 could be a new biomarker for HCC, or could possibly be used with other molecular markers to increase the specificity and sensitivity of diagnostic tests for HCC. Finally, restoration of PCDH10 could be a potentially valuable therapeutic target for HCC therapy.

\section{References}

1. Chen W, Zheng R, Baade PD, Zhang S, Zeng H, Bray F, Jemal A, Yu XQ and He J: Cancer statistics in China, 2015. CA Cancer J Clin 66: 115-132, 2016.

2. Siegel RL, Miller KD and Jemal A: Cancer statistics, 2016. CA Cancer J Clin 66: 7-30, 2016.

3. Block TM, Mehta AS, Fimmel CJ and Jordan R: Molecular viral oncology of hepatocellular carcinoma. Oncogene 22: 5093-5107, 2003.

4. Nakano M, Tanaka M, Kuromatsu R, Nagamatsu H, Tajiri N, Satani M, Niizeki T, Aino H, Okamura S, Iwamoto $\mathrm{H}$, et al; Kurume Liver Cancer Study Group of Japan: Sorafenib for the treatment of advanced hepatocellular carcinoma with extrahepatic metastasis: A prospective multicenter cohort study. Cancer Med 4: 1836-1843, 2015.

5. Harimoto N, Shirabe K, Ikegami T, Yoshizumi T, Maeda T, Kajiyama K, Yamanaka $\mathrm{T}$ and Maehara Y: Postoperative complications are predictive of poor prognosis in hepatocellular carcinoma. J Surg Res 199: 470-477, 2015.

6. Nollet F, Kools P and van Roy F: Phylogenetic analysis of the cadherin superfamily allows identification of six major subfamilies besides several solitary members. J Mol Biol 299: 551-572, 2000.

7. Halbleib JM and Nelson WJ: Cadherins in development: Cell adhesion, sorting, and tissue morphogenesis. Genes Dev 20: 3199-3214, 2006.

8. Redies C, Vanhalst K and Roy F: delta-Protocadherins: Unique structures and functions. Cell Mol Life Sci 62: 2840-2852, 2005.

9. Morishita $\mathrm{H}$ and Yagi T: Protocadherin family: Diversity, structure, and function. Curr Opin Cell Biol 19: 584-592, 2007.

10. Yu JS, Koujak S, Nagase S, Li CM, Su T, Wang X, Keniry M, Memeo L, Rojtman A, Mansukhani M, et al: PCDH8, the human homolog of PAPC, is a candidate tumor suppressor of breast cancer. Oncogene 27: 4657-4665, 2008.

11. Hu X, Sui X, Li L, Huang X, Rong R, Su X, Shi Q, Mo L, Shu X, Kuang Y, et al: Protocadherin 17 acts as a tumour suppressor inducing tumour cell apoptosis and autophagy, and is frequently methylated in gastric and colorectal cancers. J Pathol 229: 62-73, 2013.

12. Yin X, Xiang T, Mu J, Mao H, Li L, Huang X, Li C, Feng Y, Luo X, Wei Y, et al: Protocadherin 17 functions as a tumor suppressor suppressing Wnt/ $\beta$-catenin signaling and cell metastasis and is frequently methylated in breast cancer. Oncotarget 7: 51720-51732, 2016.

13. Imoto I, Izumi H, Yokoi S, Hosoda H, Shibata T, Hosoda F, Ohki M, Hirohashi S and Inazawa J: Frequent silencing of the candidate tumor suppressor PCDH20 by epigenetic mechanism in non-small-cell lung cancers. Cancer Res 66: 4617-4626, 2006.

14. Chen T, Long B, Ren G, Xiang T, Li L, Wang Z, He Y, Zeng Q, Hong $\mathrm{S}$ and $\mathrm{Hu}$ G: Protocadherin 20 acts as a tumor suppressor gene: Epigenetic inactivation in nasopharyngeal carcinoma. J Cell Biochem 116: 1766-1775, 2015.

15. Morrow EM, Yoo SY, Flavell SW, Kim TK, Lin Y, Hill RS, Mukaddes NM, Balkhy S, Gascon G, Hashmi A, et al: Identifying autism loci and genes by tracing recent shared ancestry. Science 321: 218-223, 2008 .

16. Li Z, Chim JC, Yang M, Ye J, Wong BC and Qiao L: Role of PCDH10 and its hypermethylation in human gastric cancer. Biochim Biophys Acta 1823: 298-305, 2012.

17. Zhong X, Zhu Y, Mao J,Zhang J and Zheng S: Frequent epigenetic silencing of PCDH10 by methylation in human colorectal cancer. J Cancer Res Clin Oncol 139: 485-490, 2013.

18. Tang X, Yin X, Xiang T, Li H, Li F, Chen L and Ren G: Protocadherin 10 is frequently downregulated by promoter methylation and functions as a tumor suppressor gene in non-small cell lung cancer. Cancer Biomark 12: 11-19, 2012-2013.

19. Qiu C, Bu X and Jiang Z: Protocadherin-10 acts as a tumor suppressor gene, and is frequently downregulated by promoter methylation in pancreatic cancer cells. Oncol Rep 36: 383-389, 2016.

20. Fang S, Huang SF, Cao J, Wen YA, Zhang LP and Ren GS: Silencing of PCDH10 in hepatocellular carcinoma via de novo DNA methylation independent of HBV infection or HBX expression. Clin Exp Med 13: 127-134, 2013.

21. Deng QK, Lei YG, Lin YL, Ma JG and Li WP: Prognostic value of protocadherin10 (PCDH10) methylation in serum of prostate cancer patients. Med Sci Monit 22: 516-521, 2016. 
22. Yu J, Cheng YY, Tao Q, Cheung KF, Lam CN, Geng H, Tian LW, Wong YP, Tong JH, Ying JM, et al: Methylation of protocadherin 10, a novel tumor suppressor, is associated with poor prognosis in patients with gastric cancer. Gastroenterology 136 : 640-651.e1, 2009.

23. Li M, Yan DG and Liu JL: Methylation status of PCDH10 and RASSF1A gene promoters in colorectal cancer. Zhonghua Yi Xue Za Zhi 96: 456-459, 2016 (In Chinese).

24. Jao TM, Tsai MH, Lio HY, Weng WT, Chen CC, Tzeng ST, Chang CY, Lai YC, Yen SJ, Yu SL, et al: Protocadherin 10 suppresses tumorigenesis and metastasis in colorectal cancer and its genetic loss predicts adverse prognosis. Int J Cancer 135: 2593-2603, 2014.

25. Nakao S, Platek A, Hirano S and Takeichi M: Contact-dependent promotion of cell migration by the OL-protocadherin-Nap1 interaction. J Cell Biol 182: 395-410, 2008.
26. Grove EA: Turning neurons into a nervous system. Development 135: 2203-2206, 2008

27. Li Z, Yang Z, Peng X, Li Y, Liu Q and Chen J: Nuclear factor- $\kappa B$ is involved in the protocadherin-10-mediated pro-apoptotic effect in multiple myeloma. Mol Med Rep 10: 832-838, 2014.

28. Xu Y, Yang Z, Yuan H, Li Z, Li Y, Liu Q and Chen J: PCDH10 inhibits cell proliferation of multiple myeloma via the negative regulation of the Wnt $/ \beta$-catenin/BCL-9 signaling pathway. Oncol Rep 34: 747-754, 2015.

29. Hers I, Vincent EE and Tavaré JM: Akt signalling in health and disease. Cell Signal 23: 1515-1527, 2011.

30. Bozulic L and Hemmings BA: PIKKing on PKB: Regulation of PKB activity by phosphorylation. Curr Opin Cell Biol 21: 256-261, 2009.

31. Manning BD and Cantley LC: AKT/PKB signaling: Navigating downstream. Cell 129: 1261-1274, 2007. 